\title{
97. Note on Left Simple Semigroups
}

By Tôru SAITô

Tokyo Gakugei University

(Comm. by K. ShodA, M.J.A., Oct. 12, 1959)

1. Teissier [5] considered homomorphisms of a left simple semigroup with no idempotent onto a semigroup which contains at least one idempotent, and characterized the inverse images of idempotents in such homomorphic mappings. In this note, we consider a method of constructing such a homomorphism, which turns out to be the finest in such homomorphisms.

We use terminologies in [2] without definitions and use the results obtained in [2] and [3] freely.

2. In this note, we denote a left simple semigroup by $S$.

In $S$, we define a binary relation ${ }_{s} \Sigma$ as follows:

for $a, b \in S, a \equiv b\left({ }_{S} \Sigma\right)$ means that there exists a finite sequence of elements $m_{1}, \cdots, m_{n-1}$ such that

$$
a S \gamma m_{1} S \gamma \cdots \gamma m_{n-1} S \gamma b S \text {, }
$$

where $x S \curlyvee y S$ signifies that the sets $x S$ and $y S$ have at least one element in common.

It is easy to see that ${ }_{S} \Sigma$ is an equivalence relation in $S$, which is left regular, that is,

$$
a \equiv b\left({ }_{s} \Sigma\right) \text { implies } c a \equiv c b\left({ }_{s} \Sigma\right) \text {. }
$$

In Dubreil's terminology, ${ }_{s} \Sigma$ is the generalized left reversible equivalence associated to $S[1$, p. 258].

Lemma 1. $a c \equiv a\left({ }_{s} \Sigma\right)$ for all $a, c \in S$ (cf. [1, p. 260, Théorème 8]).

Proof. For any $s \in S$, we have $(a c) s=a(c s)$. Hence we have $a c S \gamma a S$, and so $a c \equiv a\left({ }_{S} \Sigma\right)$.

Lemma 2. ${ }_{s} \Sigma$ is an equivalence relation which is regular.

Proof. It suffices to show that ${ }_{s} \Sigma$ is right regular, that is, $a \equiv b\left({ }_{s} \Sigma\right)$ implies $a c \equiv b c\left({ }_{s} \Sigma\right)$. And, in fact, if $a \equiv b\left({ }_{s} \Sigma\right)$, then, by Lemma 1, we have $a c \equiv a \equiv b \equiv b c\left({ }_{S} \Sigma\right)$.

Now, we denote the core of $S$ by $I . \quad I$ is a normal and left unitary subsemigroup of $S$ [2, Theorem 1$]$.

Lemma 3. For any $a \in S$, there exists an element $i \in I$ such that $a \equiv i\left({ }_{s} \Sigma\right)$.

Proof. Since $S$ is left simple, we can take an element $u$ such that $u a=a$. $u$ is clearly an element of $I$, and also, by Lemma 1 , we have $a=u a \equiv u\left({ }_{s} \Sigma\right)$.

Since ${ }_{s} \Sigma$ is a regular equivalence relation in $S,{ }_{s} \Sigma$ induces a regular equivalence relation in semigroup $I$. Hence we can consider 
the quotient semigroup $I /{ }_{s} \Sigma$ of the semigroup $I$. We denote the totality of classes which are the elements of $I / /_{s} \Sigma$ by $\left\{J_{\lambda} ; \lambda \in \Lambda\right\}$, and denote the product of $J_{\lambda}$ and $J_{\mu}$ in $I /_{S} \Sigma$ by $J_{\lambda} \cdot J_{\mu}$.

Lemma 4. $J_{\lambda} \cdot J_{\mu}=J_{\lambda}$ for all $J_{\lambda}, J_{\mu} \in I / s_{s}$.

Proof. For $j \in J_{\lambda}, j^{\prime} \in J_{\mu}, J_{\lambda} \cdot J_{\mu}$ is, by definition, the class which contains the element $j j^{\prime}$. On the other hand, by Lemma 1 , we have $j j^{\prime} \equiv j\left({ }_{s} \Sigma\right)$, and so $j j^{\prime}$ belongs to the class which contains $j$, that is, belongs to $J_{\lambda}$. Hence $J_{\lambda} \cdot J_{\mu}=J_{\lambda}$.

Now, we consider a mapping $\varphi$ of $S$ into $I /{ }_{s} \Sigma$. For any $a \in S$, $\varphi(a)$ is defined to be the class $J_{\lambda}$ which contains an element $i$ such that $i \equiv a\left({ }_{s} \Sigma\right)$. By Lemma 3, $\varphi(a)$ is defined certainly for all $a \in S$, and it is clear that $\varphi(a)$ is uniquely determined irrespective of the choice of element $i$.

Lemma 5. $\varphi(a)$ is a homomorphism of $S$ onto $I /{ }_{S} \Sigma$.

Proof. For $a, b \in S$, we take an element $i$ such that $a \equiv i\left({ }_{s} \Sigma\right)$. Then, by Lemma 1, we have $\left.a b \equiv a \equiv i{ }_{(} \Sigma\right)$, and so $\varphi(a b)=\varphi(a)$. Hence, by Lemma 4 , we have $\varphi(a b)=\varphi(a)=\varphi(a) \cdot \varphi(b)$, and so $\varphi$ is a homomorphism. On the other hand, for any $J_{\lambda} \in I /{ }_{s} \Sigma$, we take an element $j \in J_{\lambda}$. Then, by definition, $\varphi(j)=J_{\lambda}$, and so $\varphi$ is a homomorphism onto $I / s \Sigma$.

In [2], we proved that, for a left simple semigroup $S$, there exists a homomorphism $\theta$ of $S$ onto a group $G$ such that the kernel of $\theta$ is $I$ [2, Theorem 3].

Lemma 6. If $\theta(a)=\theta(b)$ and $x a=b$, then $x \in I$.

Proof. Under the assumption of this lemma, we have $\theta(x) \theta(a)$ $=\theta(b)=\theta(a)$, and so $\theta(x)=e$, where $e$ is the identity element of group $G$. Therefore we have $x \in I$.

Now, we consider the direct product $T$ of $I /{ }_{S} \Sigma$ and the group $G$ above-mentioned. And we define a mapping $\psi$ of $S$ into $T$ as follows: $\psi(a)=(\varphi(a), \theta(a))$.

$\psi$ is evidently a homomorphism of $S$ into $T$.

Lemma 7. An element $\left(J_{2}, g\right)$ of $T$ is an idempotent, if and only if $g$ is the identity element $e$ of $G$.

Proof. By Lemma 4, we have $\left(J_{\lambda}, g\right)\left(J_{\lambda}, g\right)=\left(J_{\lambda} \cdot J_{\lambda}, g^{2}\right)=\left(J_{\lambda}, g^{2}\right)$. Hence $\left(J_{\lambda}, g\right)$ is an idempotent, if and only if $g^{2}=g$, and so if and only if $g=e$.

Lemma 8. $\quad \psi^{-1}\left(J_{\lambda}, e\right)=J_{\lambda}$.

Proof. For any $j \in J_{\lambda}$, we have $\varphi(j)=J_{\lambda}$. Also, since $j \in J_{\lambda} \subseteq I$, we have $\theta(j)=e$. Hence we have $\psi(j)=\left(J_{\lambda}, e\right)$, and so $J_{\lambda} \subseteq \psi^{-1}\left(J_{\lambda}, e\right)$. Conversely, let us suppose that $\psi(a)=\left(J_{\lambda}, e\right)$. Then, since $\theta(a)=e$, we have $a \in I$. Hence $a \in J_{\mu}$ for some $\mu \in \Lambda$. But then, we have $\varphi(a)=J_{\mu}$ and $\psi(a)=\left(J_{\mu}, e\right)$. By assumption, we have $\psi(a)=\left(J_{\lambda}, e\right)$ and so $a \in J_{\mu}$ $=J_{\lambda}$. Hence $\psi^{-1}\left(J_{\lambda}, e\right) \subseteq J_{\lambda}$. 
Lemma 9. $\psi$ is a homomorphism onto $T$.

Proof. Let $\left(J_{\lambda}, g\right)$ be an arbitrary element of $T$. Since $\theta$ is a homomorphism onto $G$, there exists an element $a$ such that $\theta(a)=g$. Also we take any element $j \in J_{\lambda}$. Then, by Lemma 8 , we have $\psi(j)$ $=\left(J_{\lambda}, e\right)$. Hence we have $\psi(j a)=\psi(j) \psi(a)=\left(J_{\lambda}, e\right)(\varphi(a), g)=\left(J_{\lambda} \cdot \varphi(a), e g\right)$ $=\left(J_{\lambda}, g\right)$.

Summarizing the above lemmas, we obtain the following

Theorem 1. $\psi$ is a homomorphism of a left simple semigroup $S$ onto a semigroup $T$ which contains at least one idempotent, and in this homomorphism $\psi$, the inverse images of idempotents of $T$ coincide with the classes $J_{\lambda}$ which are the elements of $I /_{s} \Sigma$.

3. Now we consider a homomorphism $\psi^{*}$ of left simple semigroup $S$ onto a semigroup $T^{*}$ which contains at least one idempotent. Being a homomorphic image of a left simple semigroup, $T^{*}$ is also left simple. And it is well known that left simple semigroup $T^{*}$ with idempotent can be represented by the direct product of two semigroups $U^{*}$ and $G^{*}$, where $U^{*}$ is a right anti-semigroup in terminology of Thierrin [6], that is, a semigroup which satisfies the condition as follows:

and $G^{*}$ is a group (cf. [4]).

$$
u u^{\prime}=u \quad \text { for all } u, u^{\prime} \in U^{*},
$$

In association with the homomorphism $\psi^{*}$ :

$$
\psi^{*}(a)=(u, g) \quad \text { where } a \in S, u \in U^{*}, g \in G^{*},
$$

we consider mappings $\varphi^{*}$ and $\theta^{*}$ such that

$$
\varphi^{*}(a)=u, \quad \theta^{*}(a)=g .
$$

$\varphi^{*}$ and $\theta^{*}$ are easily seen to be homomorphisms of $S$ onto $U^{*}$ and $G^{*}$ respectively.

Lemma 10. An element $(u, g)$ of $T^{*}$ is an idempotent, if and only if $g$ is the identity element $e^{*}$ of the group $G^{*}$.

Proof is similar as in Lemma 7.

Lemma 11. $\varphi^{*}(a b)=\varphi^{*}(a)$ for all $a, b \in S$.

Proof. $\varphi^{*}(a b)=\varphi^{*}(a) \varphi^{*}(b)=\varphi^{*}(a)$.

Lemma 12. $\varphi(a)=\varphi(b)$ implies $\varphi^{*}(a)=\varphi^{*}(b)$.

Proof. If $\varphi(a)=\varphi(b)$, then the elements $a$ and $b$ are congruent modulo ${ }_{s} \Sigma$ to an element of the class $\varphi(a)=\varphi(b)$, and so $a \equiv b\left({ }_{s} \Sigma\right)$. Hence there exists a finite sequence of elements $m_{1}, m_{2}, \cdots, m_{n-1}$ such that

$$
a S \gamma m_{1} S \gamma m_{2} S \gamma \cdots \gamma m_{n-1} S \gamma b S \text {. }
$$

Therefore $a s=m_{1} s_{1}$ for some $s, s_{1} \in S$. But then, we have, by Lemma 11,

Similarly, we have

$$
\varphi^{*}(a)=\varphi^{*}(a s)=\varphi^{*}\left(m_{1} s_{1}\right)=\varphi^{*}\left(m_{1}\right) \text {. }
$$

$$
\varphi^{*}\left(m_{1}\right)=\varphi^{*}\left(m_{2}\right), \cdots, \varphi^{*}\left(m_{n-1}\right)=\varphi^{*}(b),
$$

and hence we have $\varphi^{*}(a)=\varphi^{*}(b)$. 
Lemma 13. $\theta^{*}(i)=e^{*}$ for all $i \in I$.

Proof. $\theta^{*}$ is a homomorphism of $S$ onto group $G^{*}$. Hence the kernel $\left\{x ; \theta^{*}(x)=e^{*}\right\}$ contains the core $I[2$, Theorem 2]. Hence, for any $i \in I$, we have $\theta^{*}(i)=e^{*}$.

Lemma 14. $\theta(a)=\theta(b)$ implies $\theta^{*}(a)=\theta^{*}(b)$.

Proof. Let us suppose that $\theta(a)=\theta(b)$. By the left simplicity of $S$, there exists an element $x$ such that $x a=b$. Then, by Lemma 6 , we have $x \in I$. Hence, by Lemma 13, we have $\theta^{*}(x)=e^{*}$, and so we have $\theta^{*}(b)=\theta^{*}(x a)=\theta^{*}(x) \theta^{*}(a)=\theta^{*}(a)$.

Lemma 15. $\psi(a)=\psi(b)$ implies $\psi^{*}(a)=\psi^{*}(b)$.

Proof. This is an immediate consequence of Lemmas 12 and 14.

By Lemma 15, we can consider a homomorphism $\tau$ of $T$ onto $T^{*}$ such that $\psi^{*}=\tau \psi$. Thus, we obtain the following

Theorem 2. Let $\psi^{*}$ be a homomorphism of left simple semigroup $S$ onto a semigroup $T^{*}$ which contains at least one idempotent. Then, there exists a homomorphism $\tau$ of $T$ onto $T^{*}$ such that $\psi^{*}=\tau \psi$.

\section{References}

[1] P. Dubreil: Algèbre I, $2^{e}$ édi., Gauthier-Villars (1954).

[2] T. Saitô: Homomorphisms of a left simple semigroup onto a group, Proc. Japan Acad., 34, 664-667 (1958).

[3] — : Supplement to " Homomorphisms of a left simple semigroup onto a group", Proc. Japan Acad., 35, 114 (1959).

[4] Th. Skolem: Some remarks on semi-groups, Norske Vid. Selsk. Forh., 24, 4247 (1952).

[5] M. Teissier: Sur les demi-groupes ne contenant pas d'élément idempotent, C. R. Acad. Sci. Paris, 237, 1375-1377 (1953).

[6] G. Thierrin: Sur une classe de demi-groupes inversifs, C. R. Acad. Sci. Paris, 234, 177-179 (1952). 